\title{
Propuesta de variantes para la refinacion del aceite de pescado para obtener un efluente con propiedades fertilizantes
}

\section{Proposal of variants for the refining of fish oil to obtain an effluent with fertilizing properties}

Reynaldo Francisco Cherrepano Manrique', Pilar Rosario Camos Pichilingue, Yasmin Jesus Vélez Chang, Gladys Gavedia Garcia, Cinthia Sánchez Espinoza, Miguel Angel Durand Meza², Lesly Mary Soto Neyra²

\begin{abstract}
RESUMEN
Objetivo: El presente trabajo de investigación es una propuesta para proponer variantes en la refinación del aceite de pescado, donde normalmente se utiliza el hidróxido de sodio como agente neutralizante a fin de hacer precipitar los ácidos grasos libres en forma de jabones o sales formados a partir de la reacción de neutralización entre el agente alcalino y los ácidos grasos presentes en la composición del aceite crudo de pescado. Material y Métodos: Estos ácidos grasos libres se van formando espontáneamente en el almacenamiento del aceite crudo de pescado que se acelera cuando el aceite hace contacto con la humedad del ambiente $u$ otro contacto acuoso. El propósito del presente trabajo es también presentar iniciativas favorables para el medio ambiente, en este caso en lugar de obtener un efluente que se va a destinar su vertimiento a la red pública y finalmente impacta a un cuerpo de agua, se propone la obtención de un efluente con propiedades fertilizantes, cuya actuación favorable para el desarrollo vegetativo de algún cultivo se demostraría con su aplicación foliar para algún cultivo. Resultados: Se recomendaría un análisis completo de micronutrientes y macronutrientes del efluente obtenido, siendo relevante su contenido en nitrógeno, fosforo y potasio. Conclusión: Con esta variante en la etapa del proceso de purificación del aceite crudo de pescado que es la refinación del aceite se estaría reduciendo los impactos directos e indirectos ambientales que se ocasionan en la refinación industrial del aceite de pescado.
\end{abstract}

Palabras clave: Neutralización, Aceite de pescado, Efluente.

\section{ABSTRACT}

Objective: The present research work is a proposal to propose variants in the refining of fish oil, where sodium hydroxide is normally used as a neutralizing agent in order to precipitate free fatty acids in the form of soaps or salts formed from the neutralization reaction between the alkaline agent and the fatty acids present in the composition of crude fish oil. Materials and Methods: These free fatty acids are formed spontaneously in the storage of crude fish oil that accelerates when the oil comes into contact with the humidity of the environment or other aqueous contact. The purpose of this work is also to present favorable initiatives for the environment, in this case instead of obtaining an effluent that is going to be discharged to the public network and finally impacts a body of water, it is proposed to obtain a effluent with fertilizing properties, whose favorable performance for the vegetative development of some crop would be demonstrated with its foliar application for some crop. Results: A complete analysis of micronutrients and macronutrients of the effluent obtained would be recommended, its nitrogen, phosphorus and potassium content being relevant. Conclusion: With this variant in the stage of the crude fish oil purification process, which is the refining of the oil, the direct and indirect environmental impacts that are caused in the industrial refining of fish oil would be reduced.

Keywords: Neutralization, Fish oil, Effluent

\section{INTRODUCCIÓN}

Existe una evidente tendencia holística en el manejo y disposición final de los recursos, a fin de proteger y conservar el medio ambiente al respecto Vargas at el (2014) presentan una metodología para el ordenamiento territorial que integra, desde una perspectiva holística, las técnicas derivadas de la teoría de la decisión y los modelos de conocimientos sustentados en el criterio de los actores sociales de la comunidad. Ello constituye un nuevo enfoque que puede ser aplicado en cualquier parte del país para favorecer el proceso de toma de decisiones a nivel local, lograr el manejo racional de los recursos socioeconómicos y ambientales acorde a las políticas actuales de Cuba para garantizar la producción sostenible de alimentos con la cantidad y la calidad requeridas, que conlleve a la autosuficiencia alimentaria y a la reducción de importaciones.

Una opción más aplicable en los recursos, productos, efluentes, residuos sólidos derivados de la industria alimenticia, en la cual se pueden proponer cambios en los proceso y técnicas para propiciar resultados favorables al medio ambiente en este propósito señala Acosta (1999) que la evolución de las técnicas ha permitido ampliar la variedad de recursos que se puede Recibido 20/08/2021 Aprobado 29/08/2021

Este es un artículo de acceso abierto, distribuido bajo los términos de la Licencia Creative Commons Atribución 4.0 Internacional (http://creativecommons.org/licenses/by/4.0/)

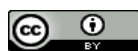

'Docente de la Facultad de Educación. Universidad Nacional José Faustino Sánchez Carrión. Huacho, Huaura, Lima, Perú

ORCID: 0000-0001-8249-2480. Email: rcherrepano@unjfsc.edu.pe

2Alumnos de la Facultad e Educación de la Universidad Nacional José Faustino Sánchez Carrión. Huacho, Huaura, Lima, Perú. 
extraer de la naturaleza, modificaciones genéticas, nuevos usos etc. La aplicación de las tecnologías que modifican los recursos naturales, puede generar impactos positivos o negativos al ambiente, por lo cual deben manejarse cuidadosamente.

Estas propuestas de cambios en las tecnologías y técnicas requieren la integración con las políticas de gobierno, a fin de que se incentiven o favorezcan el uso de algunos insumos o equipos cuyo uso genere menos impacto al medio ambiente, los productos y/ o subproductos obtenidos de la aplicación de estas modificaciones tengan el auspicio de los gobiernos locales y/o regionales para su aceptación, en relación a estas apreciaciones Enkerlin at el (1994) sostiene que la transición hacia el desarrollo sostenible exigirá cambios importantes, y tal vez sacrificios en el corto plazo. Estos cambios y sacrificios se podrán justificar por el sólo hecho de revertir tendencias que preocupan a los líderes del mundo, y que en realidad deberían preocuparnos a todos, sobre todo a los jóvenes, los futuros líderes quienes deben delegar que se actúe con responsabilidad hacia las generaciones que recibirán este compromiso con el ambiente. Hoy en día el desarrollo sostenible se ha caracterizado por predominio de la tendencia hacia la máxima rentabilidad en cuanto al uso de los recursos naturales.

Las aplicaciones de propuestas en la industria alimentaria son más viables por la composición propia que presenta los efluentes que con algunas propuestas de cambios en un propósito ambientalista se puede lograr efluentes con algunas ventajas ambientales, en este objetivo Lee (2019) nos explica que, al enorme crecimiento de la población, inevitablemente se generan toneladas de efluentes a lo largo de las actividades agrícolas. La eliminación inadecuada de efluentes induce una contaminación perpetua de las fuentes de agua del mar y del río, lo que posteriormente ha planteado la preocupación ambiental pública. Por esa razón, el protocolo de manejo de efluentes agrícolas fue señalado como un área de interés para la investigación. A pesar de los peligros ambientales, los efluentes agrícolas tienen el potencial de transformarse de desechos en riqueza a través de procesos biológicos, fisicoquímicos, termoquímicos o una combinación de procesos.

Las mismas características de los efluentes de las almazaras de aceite de palma (POME) y de las aguas residuales de las almazaras (OMW) hacen posible el tratamiento de estos residuos utilizando el método de tratamiento similar. En general, el tratamiento biológico requiere un tiempo de proceso más largo en comparación con las tecnologías fisicoquímicas y termoquímicas a pesar de su operación fácil y de bajo costo. Comparativamente, los métodos fisicoquímicos y termoquímicos amplían su potencialidad para convertir los efluentes agrícolas en productos de mayor valor de manera más eficiente. Este documento revisa la fuente y las características tanto de POME como de OMW. Posteriormente, se realizó una comparación de los tratamientos actuales y alternativos para ambos efluentes antes de que se pavimenten las perspectivas futuras del tratamiento de ambos efluentes en función del bienestar humano, ambiental y económico.

Con respecto a este orden de ideas, sobre la recuperación o reciclo de las aguas residuales proedenres de la industria aceitera, Althausen (2016) nos explica que las aguas residuales de plantas de beneficio de aceite de palma o Palm Oil Mill Effluent (pome, por su sigla en inglés), requieren tratamiento antes de su uso en riego o su descarga en aguas superficiales. Al mismo tiempo, un tratamiento moderno de pome puede convertir la alta carga orgánica en biogás, una mezcla de gases con un valor energético de $6 \mathrm{kWh} / \mathrm{m}$

\section{MATERIALES Y MÉTODOS}

\section{Población}

Está compuesto por efluentes vertidos de la unidad de refinación de la planta industria que utiliza aceite de pescado (anchoveta) como materia prima. Efluente involucra el agua de lavado del aceite refinado y también el agua acida de recuperación de los ácidos grasos de las sales formadas en la refinación del aceite.

\section{Muestra}

Para la toma de muestras se realizaron los siguientes procedimientos se tomó muestras cuando se utiliza el agente neutralizante hidróxido de sodio e hidróxido de potasio. El volumen de las muestras para cada caso 2 litros de efluente.

\section{Procedimientos}

Los procedimientos se desarrollaron de la siguiente manera:

- Recolecta de muestras de efluentes obtenidos en la refinación del aceite a nivel laboratorio.

- $\quad$ Evaluación de las dos alternativas de refinación de aceite, haciendo los experimentos en laboratorio utilizando el hidróxido de sodio y el hidróxido de potasio.

Análisis físico-químico comparativo de las muestras del agua de lavado y el agua de recuperación de los ácidos grasos a partir de los jabones formados en la neutralización del aceite de pescado.

- Tratamiento y análisis de los efluentes acuosos obtenidos de la refinación del aceite, utilizando los dos tipos de agentes neutralizantes.

- $\quad$ Presentación de datos y resultados del trabajo de investigación.

\section{RESULTADOS}

Los resultados de Análisis Físico-Químico de la mezcla de agua de lavado del aceite refinado con el agua acida resultante del tratamiento de las sales formadas para formar ácidos grasos y la glicerina. 
A esta mezcla de resultantes acuosos se le realizo el análisis en el propósito de investigar sus propiedades fertilizantes, para el caso que se utilizó como agente neutralizante el hidróxido de sodio $(\mathrm{NaOH})$ y para la otra prueba experimental donde se propone la utilización del hidróxido de potasio $(\mathrm{KOH})$. Generándose la tabla 1 y la tabla 2 respectivamente.

\section{Tabla 1}

Análisis físico-químico del efluente resultante de la refinación con $\mathrm{KOH}$.

\begin{tabular}{ll}
\hline $\begin{array}{l}\text { Parámetro Físico } \\
\text { Químico }\end{array}$ & Resultado \\
\hline Ph & 8.24 \\
\hline $\mathrm{C} . \mathrm{E}(\mathrm{mS} / \mathrm{cm})$ & 4.71 \\
\hline Sólidos totales $(\mathrm{g} / \mathrm{L})$ & 3.59 \\
\hline $\mathrm{M} . \mathrm{O} .(\mathrm{mg} / \mathrm{L})$ & 722 \\
\hline $\mathrm{N}(\mathrm{mg} / \mathrm{L})$ & 25760 \\
\hline $\mathrm{P}(\mathrm{mg} / \mathrm{L})$ & 88.86 \\
\hline $\mathrm{K}(\mathrm{mg} / \mathrm{L})$ & 1707.51 \\
\hline $\mathrm{Ca}(\mathrm{mg} / \mathrm{L})$ & 17.19 \\
\hline $\mathrm{Mg}(\mathrm{mg} / \mathrm{L})$ & 1.85 \\
\hline $\mathrm{C} / \mathrm{N}$ & 0.03 \\
\hline $\mathrm{Fe} \mathrm{ppm}$ & 0.055 \\
\hline $\mathrm{Zn} \mathrm{ppm}$ & 0.58 \\
\hline $\mathrm{Cu} \mathrm{ppm}$ & 0.005 \\
\hline $\mathrm{Mn} \mathrm{ppm}$ & 0.11 \\
\hline
\end{tabular}

Fuente: INIA

\section{Tabla 2}

Análisis físico-químico del efluente resultante de la refinación con $\mathrm{NaOH}$

\begin{tabular}{ll}
\hline $\begin{array}{l}\text { Parámetro } \\
\text { Químico }\end{array}$ & Resultado- \\
\hline $\mathrm{Ph} \quad$ & 9.29 \\
\hline $\mathrm{C} . \mathrm{E}(\mathrm{mS} / \mathrm{cm})$ & 323.00 \\
\hline Sólidos totales $(\mathrm{g} / \mathrm{L})$ & 1.70 \\
\hline $\mathrm{M} . \mathrm{O} .(\mathrm{mg} / \mathrm{L})$ & 1215 \\
\hline $\mathrm{N}(\mathrm{mg} / \mathrm{L})$ & 560 \\
\hline $\mathrm{P}(\mathrm{mg} / \mathrm{L})$ & 82.58 \\
\hline $\mathrm{K}(\mathrm{mg} / \mathrm{L})$ & 201.20 \\
\hline $\mathrm{Ca}(\mathrm{mg} / \mathrm{L})$ & 14.08 \\
\hline $\mathrm{Mg}(\mathrm{mg} / \mathrm{L})$ & 14.03 \\
\hline $\mathrm{C} / \mathrm{N}$ & 1.26 \\
\hline $\mathrm{Fe} \mathrm{ppm}$ & 0.01025 \\
\hline $\mathrm{Zn} \mathrm{ppm}$ & 0.39 \\
\hline $\mathrm{Cu} \mathrm{ppm}$ & 0.001 \\
\hline $\mathrm{Mn} \mathrm{ppm}$ & 0.09 \\
\hline
\end{tabular}

Fuente: INIA

\section{DISCUSIÓN}

En la tabla 1 con respecto a la tabla 2 se observa una ventaja porcentual en los contenidos de los principales macronutrientes para el desarrollo vegetativo de las plantas, en este caso son el nitrógeno, fosforo y potasio, en este caso se evidencia el efecto favorable de haber utilizado el hidróxido de potasio en lugar del hidróxido de sodio, a su vez ocasionó un efecto positivo el a haber utilizado el hidróxido de amonio para neutralizar la acidez generada por ácido sulfúrico para desdoblar las sales de jabón formadas en ácidos grasos y glicerina.

En relación a los porcentajes en los contenidos de micronutrientes se aprecia valores superiores en la mayoría de los elementos químicos cunado se utilizó como agente neutralizante el hidróxido de potasio.

Por lo que se aprecia los contenidos de macronutrientes y micronutrientes del efluente obtenido según se muestra en las tablas 1 y 2 , producto obtenido al hacer un cambio en la etapa de refinación del aceite de pescado donde normalmente se utiliza el hidróxido de sodio y se propone la utilización del hidróxido de potasio, se hace evidente la proyección de utilizarse estas agua como soluciones fertilizantes para cultivos, en esta proyección se tiene el aporte de Legua (2001) explica que en sus resultados de la evaluación de crecimiento y desarrollo del rabanito, lechuga y acelga se ilustran en las Tablas 8, 9 y 10, donde se llevó a contrastación los medios de cultivos fertilizados con soluciones nutrientes a diferente relación dilución, según la Tabla 11 y al medio de cultivo testigo sin fertilización, en todos los medios de cultivo se deposita semillas para observar su germinación y crecimiento.

La respuesta de las plantas rabanito, lechuga y acelga es evidentemente favorable en los medios de cultivo que fueron fertilizados mas no así en el medio de cultivo testigo, los resultados favorables son más marcados en el caso del rabanito y lechuga. Por otro lado, se observa también un mejor resultado en el tamaño de la planta y peso fresco de tallos, hojas y ralbes. Para la relación de dilución 1:22, al menos esto es evidente en el caso del rabanito y lechuga.

\section{CONCLUSIONES}

- $\quad$ Al realizar el cambio de agente neutralizante a nivel laboratorio en la refinación de una muestra de aceite de pescado no se observó ningún inconveniente en el lavado del aceite para su remoción de restos de hidróxido de potasio de la masa de aceite, al igual que resulta cuando se neutraliza con hidróxido de sodio.

- La propuesta de aplicación de neutralizar con hidróxido de potasio es viable técnicamente pero económicamente no por el mayor costo que tiene el hidróxido de potasio con respecto al hidróxido de sodio.

De acuerdo a los resultados de análisis de laboratorio que se muestran en las tablas 1 y 2 , se evidencia el mayor contenido de macronutrientes y micronutrientes en el agua de mezcla resultante de aguas de lavado del aceite y el agua resultante en la recuperación de los ácidos grasos de las sales de jabón 
formadas en la refinación del aceite.

- Un macronutriente muy importante para el desarrollo vegetativo de un cultivo que en ese caso es el nitrógeno, su contenido en el agua resultante utilizando el hidróxido de potasio es de $25,760 \mathrm{mg} / \mathrm{L}$, mientras que cuando se neutraliza con hidróxido de sodio registra un contenido en el agua de lavado de $560 \mathrm{mg} / \mathrm{L}$. Esta gran diferencia en el contenido de nitrógeno proyecta resultados favorables al utilizarse este producto como fertilizante foliar.

- De acuerdo a los resultados obtenidos por Legua (2001) la utilización de estas aguas recuperadas de efluentes que ser vierten al sistema de alcantarillado y por ende a los cuerpos de aguas por parte de la industria aceitera, se verificó una favorable respuesta en los cultivos de rabanito, lechuga y acelga, al utilizarse estas aguas como fertilizantes.

\section{BIBLIOGRAFÍA}

Acosta, A. (1999). Propuestas de la Tecnología Limpia en la industrialización de los Recursos Naturales. En: Primer simposium Internacional de Industrialización de los Recursos Naturales pare el Desarrollo Nacional. Ed. Colegio de Ingenieros; del Perú, Capítulo de Ingeniería Química. Lima.
Althausin, M. (2016). Tratamiento de efluentes de la planta de beneficio-convertir un residuo en un recurso. Revista Palmas. Vol. 37, N Especial. pp 31-37.

Enkerlin, E., Rodríguez, S. y Cano, G. (1997). El desarrollo sostenible ¿un nuevo paradigma?. En: Ciencia ambiental y desarrollo sostenible. Ed. International Thomson Editores, México.

Vargas, H.; Ponce de León, D.; Ojeda, R y Torres, F.(2014) Metodología para el uso y manejo social del recurso tierra como contribución al desarrollo local sostenible. Vol $1 \mathrm{~N}^{\circ}$.

Lee, Z. (2019) Tecnologías de tratamiento de efluentes de molinos de aceite de palma (POME) y aguas residuales de molinos de olivo (OMW). Tecnología e Innovación Ambiental. Vol 15. 100377.

Legua, J. (2001). Reducción de la contaminación en la refinación del aceite. Revista del Instituto de Investigación Facultad de Ingeniería Geológica, Minera, Metalúrgica y Geografía. Vol 4,Nº 8 UNMSM 